\title{
Rational Solutions of a Weakly Coupled Nonlocal Nonlinear Schrödinger Equation
}

\author{
Huijuan Zhou, ${ }^{1}$ Chuanzhong Li ${ }^{\mathbb{D}},{ }^{1}$ and Yueh-Lung Lin ${ }^{2}$ \\ ${ }^{1}$ Department of Mathematics, Ningbo University, Ningbo 315211, China \\ ${ }^{2}$ School of Mathematical Sciences, University of Nottingham, Ningbo 315100, China \\ Correspondence should be addressed to Chuanzhong Li; lichuanzhong@nbu.edu.cn
}

Received 18 May 2018; Accepted 17 September 2018; Published 14 October 2018

Academic Editor: Antonio Scarfone

Copyright (c) 2018 Huijuan Zhou et al. This is an open access article distributed under the Creative Commons Attribution License, which permits unrestricted use, distribution, and reproduction in any medium, provided the original work is properly cited.

\begin{abstract}
In this article, we investigate an integrable weakly coupled nonlocal nonlinear Schrödinger (WCNNLS) equation including its Lax pair. Afterwards, Darboux transformation (DT) of the weakly coupled nonlocal NLS equation is constructed, and then the degenerated Darboux transformation can be got from Darboux transformation. Applying the degenerated Darboux transformation, the new solutions $\left(q^{[1]}, r^{[1]}\right)$ and self-potential function $\left(V^{[1]}\right)$ are created from the known solutions $(q, r)$. The $\left(q^{[1]}, r^{[1]}\right)$ satisfy the parity-time (PT) symmetry condition, and they are rational solutions with two free phase parameters of the weakly coupled nonlocal nonlinear Schrödinger equation. From the plots of solutions, the compression effects of the real refractive index profile and the gain-or-loss distribution are produced.
\end{abstract}

\section{Introduction}

A nonlocal nonlinear Schrödinger (NLS) equation was recently found and shown to be an integrable infinite dimensional Hamiltonian equation. Unlike the local case, the nonlinearly induced potential is PT-symmetric [1]; thus the nonlocal NLS equation is also PT-symmetric. According to the work of Bender and Boettcher [2], the PT-symmetry plays a vital role in the spectrum of the Hamiltonian. They proved that a broad class of non-Hermitian Hamiltons [3] with PTsymmetry have real and positive spectrum; the work drew attention for several researchers who study PT-symmetry in quantum mechanics [4-7]. For a non-Hermitian Hamiltonian $\mathbf{H}=d^{2} / d x^{2}+V(x)$, it is PT-symmetric when $V(x)=V(-x)^{*}$. Under this condition, the Schrödinger equation $i \Psi_{t}=\mathrm{H} \Psi$ is PT-symmetric. It has been shown that optics can provide a good condition for testing the theory of PT-symmetry or observing the phenomenon when the PTsymmetry is broken [8-11].

As is well known, the nonlinear Schrödinger equation

$$
q_{t}(x, t)-i q_{x x}(x, t)-2 i|q(x, t)|^{2} q(x, t)=0,
$$

which is PT-symmetric, received an extensive study since the work by Shabat and Zakharov [12]. The NLS equation has many important physical applications, such as water wave [13], nonlinear optics [14], plasma physics [15], and so on.

Recently, Musslimani and Ablowitz [16] considered the solution of the nonlocal NLS equation,

$$
\begin{aligned}
& q_{t}(x, t)-i q_{x x}(x, t) \pm 2 i V(x, t) q(x, t)=0, \\
& V(x, t)=q(x, t) q^{*}(-x, t) .
\end{aligned}
$$

We first recall that the nonlocal focusing NLS equation

$$
q_{t}(x, t)-i q_{x x}(x, t)+2 i V(x, t) q(x, t)=0,
$$

from zero background by using the IST method, which implied the PT-symmetry, in other words, $x \longrightarrow-x$, $t \longrightarrow-t$ and the complex conjugation of the field envelope. Here $q(x, t)$ and $V(x, t)$ (called the PT-symmetric potential) imply the electric field envelope of the optical beam and complex refractive index distribution or an optical potential, respectively $[7,8]$, and the asterisk denotes the complex conjugation. In optics, the real part of $V(x, t)$ defined by $V_{R}(x, t)$ 
indicates the refractive index profile, and the imaginary part of $V(x, t)$ represented by $V_{I}(x, t)$ denotes the gain or loss [17] distribution. Based on $V_{R}(x, t)=V_{R}(-x, t)$ and $V_{I}(x, t)=$ $-V_{I}(-x, t)$, a PT-symmetric system can be designed.

Nevertheless, the nonlocal property of the soliton equations is not new, for example, nonlocal symmetry [18]. In this purpose, we consider another new nonsymmetric coupled nonlocal NLS equation called weakly coupled nonlocal NLS equation which is as follows:

$$
\begin{aligned}
& q_{t}(x, t)-i q_{x x}(x, t)+2 i V(x, t) q(x, t)=0, \\
& r_{t}(x, t)-i r_{x x}(x, t)+2 i q(x, t)^{2} r^{*}(-x, t) \\
& \quad+4 i V(x, t) r(x, t)=0
\end{aligned}
$$

$$
V(x, t)=q(x, t) q^{*}(-x, t) .
$$

The concept of PT-symmetry, based on the non-Hermitian Hamiltonians $[2,19-22]$, has recently attracted much attention [23], in particular in the fields of optics and photonics $[9,24,25]$. This concept offers a fertile ground for PT-related notions and experiments. Furthermore, applying such idea for the design of photonic devices has opened up many new possibilities, which allow a controlled interplay between gain and loss. The loss is abundant in physical systems but is typically considered as a problem. The gain, however, as afforded by lasers, is valuable in optoelectronics because it provides means to overcome loss [26]. Several studies have shown that the PT-symmetric optical structures can exhibit particular properties that are otherwise unattainable in traditional Hermitian structures, for example, the possibility for breaking this symmetry through an abrupt phase transition $[11,27]$, the unidirectional invisibility [28], and so on. For a few overview papers in the area of linear and nonlinear PTsymmetric systems, see [29-31].

If a Hamiltonian is PT-symmetric, there are two possibilities: Either the eigenvalues are entirely real, in which case the Hamiltonian is said to be in an unbroken PTsymmetric phase, or else the eigenvalues are partly complex and partly real, in which case the Hamiltonian is said to be in a broken PT-symmetric phase. Moreover, there is a PT-symmetry-breaking threshold, where the transition is between broken and unbroken symmetry. At this point, the behavior of Hamiltonians becomes even more interesting. In this paper, the solutions of (4) at the threshold value of eigenvalues are derived by degenerated DT. It is found that the solutions possess a similar structure with the second-order soliton, but both of them are different because the asymptotic amplitudes at two orbits for our rational solutions depend on each other and there does not exist any phase shift after the interaction. Besides, we consider the analyticity related to the phase parameters and classify the solution according to its asymptotic amplitudes on the phase parameters. We have provided the rational solutions $\left(q^{[1]}, r^{[1]}\right)$ from the weakly nonlocal NLS equation, and the analytic properties of $\left(q^{[1]}, r^{[1]}\right)$ are proved in detail.

This paper is arranged as follows: In Section 2, we construct a weakly coupled nonlocal NLS equation and its Lax representation. In Section 3, we calculate the Darboux transformation of the weakly coupled nonlocal NLS equation. In Section 4, we consider the rational solutions of the weakly coupled nonlocal NLS equation. Please see Section 5 for the conclusion.

\section{Weakly Coupled Nonlocal NLS Equation and Its Lax Representation}

The weakly coupled nonlocal NLS equation (4) can be expressed by the compatibility condition of the following Lax pair:

$$
\begin{aligned}
& \Phi_{x}(x, t ; \lambda)=M(x, t ; \lambda) \Phi(x, t ; \lambda), \\
& \Phi_{t}(x, t ; \lambda)=N(x, t ; \lambda) \Phi(x, t ; \lambda),
\end{aligned}
$$

where $\Phi(x, t ; \lambda)$ is a matrix valued function, $\lambda \in \mathbb{C}$ is the spectral parameter, and

$$
\begin{aligned}
& M(x, t ; \lambda) \\
& =\left(\begin{array}{cc}
-i \lambda E & q(x, t) E+r(x, t) \Gamma \\
q^{*}(-x, t) E+r^{*}(-x, t) \Gamma & i \lambda E
\end{array}\right), \\
& N(x, t ; \lambda)=\left(\begin{array}{ll}
N_{1} & N_{2} \\
N_{3} & N_{4}
\end{array}\right),
\end{aligned}
$$

where

$$
\begin{aligned}
N_{1}= & \left(-2 i \lambda^{2}-i q(x, t) q^{*}(-x, t)\right) E \\
& -i\left(q(x, t) r^{*}(-x, t)+q^{*}(-x, t) r(x, t)\right) \Gamma, \\
N_{2}= & \left(2 \lambda q(x, t)+i q_{x}(x, t)\right) E \\
& +\left(2 \lambda r(x, t)+i r_{x}(x, t)\right) \Gamma, \\
N_{3}= & \left(2 \lambda q^{*}(-x, t)-i q_{x}^{*}(-x, t)\right) E \\
& +\left(2 \lambda r^{*}(-x, t)-i r_{x}^{*}(-x, t)\right) \Gamma, \\
N_{4}= & \left(2 i \lambda^{2}+i q(x, t) q^{*}(-x, t)\right) E \\
& +i\left(q(x, t) r^{*}(-x, t)+q^{*}(-x, t) r(x, t)\right) \Gamma, \\
E= & \left(\begin{array}{ll}
1 & 0 \\
0 & 1
\end{array}\right), \\
\Gamma= & \left(\begin{array}{ll}
0 & 0 \\
1 & 0
\end{array}\right) .
\end{aligned}
$$

That is, $M_{t}-N_{x}+[M, N]=0$ leads to a weakly coupled nonlocal NLS equation (4),

$$
\begin{aligned}
& q_{t}(x, t)-i q_{x x}(x, t)+2 i V(x, t) q(x, t)=0, \\
& r_{t}(x, t)-i r_{x x}(x, t)+2 i q(x, t)^{2} r^{*}(-x, t) \\
& +4 i V(x, t) r(x, t)=0, \\
& V(x, t)=q(x, t) q^{*}(-x, t) .
\end{aligned}
$$




\section{Darboux Transformation for the Weakly Coupled Nonlocal NLS Equation}

It was pointed out that the Darboux transformation is a method proposed by J. G. Darboux more than 100 years ago and it is an efficient method to generate soliton solutions of integrable equations. In this section, we would like to introduce the Darboux transformation [32, 33] and degenerated Darboux transformation for the weakly coupled nonlocal NLS equation. First, we will introduce a simple gauge transformation of spectral problems (5) as follows:

$$
\Phi^{[1]}=T \Phi \text {. }
$$

It can transform linear problems (5) into a new one

$$
\begin{aligned}
& \Phi_{x}^{[1]}=M^{[1]} \Phi^{[1]}, \\
& \Phi_{t}^{[1]}=N^{[1]} \Phi^{[1]},
\end{aligned}
$$

where $M^{[1]}, N^{[1]}$ have the same forms with $M, N$ except that the $q(x, t), r(x, t), q^{*}(-x, t), r^{*}(-x, t)$ in the matrices $M, N$ are replaced with $q^{[1]}(x, t), r^{[1]}(x, t), q^{[1] *}(-x, t), r^{[1] *}(-x, t)$ in the matrices $M^{[1]}, N^{[1]}$. It is easy to obtain the equations

$$
\begin{gathered}
M^{[1]} T=T_{x}+T M, \\
N^{[1]} T=T_{t}+T N,
\end{gathered}
$$

which lead to

$$
\begin{aligned}
& M^{[1]}=T_{x} T^{-1}+T M T^{-1}, \\
& N^{[1]}=T_{z} T^{-1}+T N T^{-1} .
\end{aligned}
$$

In general, if the transformation $T$ is a polynomial of the parameter $\lambda$, we can start from

$$
T=T_{1} \lambda+T_{0},
$$

where

$$
\begin{aligned}
& T_{1}=\left(\begin{array}{ll}
a_{10} E+a_{11} \Gamma & b_{10} E+b_{11} \Gamma \\
c_{10} E+c_{11} \Gamma & d_{10} E+d_{11} \Gamma
\end{array}\right), \\
& T_{0}=\left(\begin{array}{ll}
a_{0} E+a_{1} \Gamma & b_{0} E+b_{1} \Gamma \\
c_{0} E+c_{1} \Gamma & d_{0} E+d_{1} \Gamma
\end{array}\right) .
\end{aligned}
$$

For (13), we can get

$$
\begin{aligned}
T_{x}= & T_{1 x} \lambda+T_{0 x} \\
= & \left(\begin{array}{cc}
a_{10 x} E+a_{11 x} \Gamma & b_{10 x} E+b_{11 x} \Gamma \\
c_{10 x} E+c_{11 x} \Gamma & d_{10 x} E+d_{11 x} \Gamma
\end{array}\right) \lambda \\
& +\left(\begin{array}{cc}
a_{0 x} E+a_{1 x} \Gamma & b_{0 x} E+b_{1 x} \Gamma \\
c_{0 x} E+c_{1 x} \Gamma & d_{0 x} E+d_{1 x} \Gamma
\end{array}\right) \\
= & \left(\begin{array}{cc}
0 & -2 i b_{10} E-2 i b_{11} \Gamma \\
2 i c_{10} E+2 i c_{11} \Gamma & 0
\end{array}\right) \lambda^{2} \\
& +\left(\begin{array}{ll}
H 1 & H 2 \\
H 3 & H 4
\end{array}\right) \lambda+\left(\begin{array}{cc}
K 1 & K 2 \\
K 3 & K 4
\end{array}\right),
\end{aligned}
$$

where

$$
\begin{aligned}
& H_{1}=\left(q^{[1]}(x, t) c_{10}-b_{10} q^{*}(-x, t)\right) E+\left(r^{[1]}(x, t) c_{10}\right. \\
& \left.+q^{[1]}(x, t) c_{11}-b_{10} r^{*}(-x, t)-b_{11} q^{*}(-x, t)\right) \Gamma, \\
& H_{2}=\left(-2 i b_{0}+q^{[1]}(x, t) d_{10}-a_{10} q(x, t)\right) E+\left(-2 i b_{1}\right. \\
& +q^{[1]}(x, t) d_{11}+r^{[1]}(x, t) d_{10}-a_{10} r(x, t) \\
& \left.-a_{11} q(x, t)\right) \Gamma \\
& H_{3}=\left(2 i c_{0}+q^{[1] *}(-x, t) a_{10}-d_{10} q^{*}(-x, t)\right) E \\
& +\left(2 i c_{1}+q^{[1] *}(-x, t) a_{11}+r^{[1] *}(-x, t) a_{10}\right. \\
& \left.-d_{10} r^{*}(-x, t)-d_{11} q^{*}(-x, t)\right) \Gamma, \\
& H_{4}=\left(q^{[1] *}(-x, t) b_{10}-c_{10} q(x, t)\right) E \\
& +\left(q^{[1] *}(-x, t) b_{11}+r^{[1] *}(-x, t) b_{10}-c_{10} r(x, t)\right. \\
& \left.-c_{11} q(x, t)\right) \Gamma \text {, } \\
& K_{1}=\left(q^{[1]} c_{0}-q^{*}(-x, t) b_{0}\right) E+\left(r^{[1]}(x, t) c_{0}\right. \\
& \left.+q^{[1]}(x, t) c_{1}-r^{*}(-x, t) b_{0}-b_{1} q^{*}(-x, t)\right) \Gamma, \\
& K_{2}=\left(q^{[1]} d_{0}-a_{0} q(x, t)\right) E+\left(q^{[1]}(x, t) d_{1}\right. \\
& \left.+r^{[1]}(x, t) d_{0}-a_{0} r(x, t)-a_{1} q(x, t)\right) \Gamma, \\
& K_{3}=\left(q^{[1] *}(-x, t) a_{0}-d_{0} q^{*}(-x, t)\right) E \\
& +\left(q^{[1] *}(-x, t) a_{1}+r^{[1] *}(-x, t) a_{0}-r^{*}(-x, t) d_{0}\right. \\
& \left.-d_{1} q^{*}(-x, t)\right) \Gamma \\
& K_{4}=\left(q^{[1] *}(-x, t) b_{0}-c_{0} q(x, t)\right) E+\left(q^{[1] *}(-x, t) b_{1}\right. \\
& \left.+r^{[1] *} b_{0}-c_{0} r(x, t)-c_{1} q(x, t)\right) \Gamma \text {. }
\end{aligned}
$$

By comparing the coefficient in terms of $\lambda$, we can see

$$
\begin{aligned}
& a_{10 x}=a_{11 x}=0, \\
& b_{10}=b_{11}=0, \\
& d_{10 x}=d_{11 x}=0, \\
& c_{10}=c_{11}=0, \\
& q^{[1]}(x, t) d_{10}-2 i b_{0}-q(x, t) a_{10}=0, \\
& r^{[1]}(x, t) d_{10}+q^{[1]}(x, t) d_{11}-2 i b_{1}-q(x, t) a_{11} \\
& \quad-r(x, t) a_{10}=0 .
\end{aligned}
$$

So functions $a_{10}, a_{11}, d_{10}, d_{11}$ are independent of $x$. For $T_{t}+T N=N^{[1]} T$, we can get that functions $a_{10}, a_{11}, d_{10}$, 
$d_{11}$ should be independent of $t$ using the same method. This suggests that $a_{10}, a_{11}, d_{10}$, and $d_{11}$ are constants. Then the form of Darboux transformation operator is as follows:

$$
\begin{aligned}
T= & T_{1}(\lambda) \\
= & \left(\begin{array}{cc}
a_{10} E+a_{11} \Gamma & \mathbf{0} \\
\mathbf{0} & d_{10} E+d_{11} \Gamma
\end{array}\right) \lambda \\
& +\left(\begin{array}{cc}
a_{0} E+a_{1} \Gamma & b_{0} E+b_{1} \Gamma \\
c_{0} E+c_{1} \Gamma & d_{0} E+d_{1} \Gamma
\end{array}\right) .
\end{aligned}
$$

Now we will try to determine the specific expression of the matrix $T$. In order to solve this problem, we must use eigenfunction which contains $\lambda$ to determine their expressions through the parameterized method. Next, we will introduce the properties of eigenfunction in spectral problem, with lemma as follows.

Lemma 1. The eigenfunctions possess the following property:

$$
\begin{aligned}
& \left(\begin{array}{l}
\phi_{110}\left(x, t ; \lambda_{1}\right) E+\phi_{111}\left(x, t ; \lambda_{1}\right) \Gamma \\
\phi_{120}\left(x, t ; \lambda_{1}\right) E+\phi_{121}\left(x, t ; \lambda_{1}\right) \Gamma
\end{array}\right) \\
& =\left(\begin{array}{c}
\phi_{120}^{*}\left(-x, t ;-\lambda_{1}^{*}\right) E+\phi_{121}^{*}\left(-x, t ;-\lambda_{1}^{*}\right) \Gamma \\
-\phi_{110}^{*}\left(-x, t ;-\lambda_{1}^{*}\right) E-\phi_{111}^{*}\left(-x, t ;-\lambda_{1}^{*}\right) \Gamma
\end{array}\right),
\end{aligned}
$$

where

$$
\Phi_{1}\left(x, t ; \lambda_{1}\right)=\left(\begin{array}{l}
\phi_{110}\left(x, t ; \lambda_{1}\right) E+\phi_{111}\left(x, t ; \lambda_{1}\right) \Gamma \\
\phi_{120}\left(x, t ; \lambda_{1}\right) E+\phi_{121}\left(x, t ; \lambda_{1}\right) \Gamma
\end{array}\right)
$$

is an eigenfunction associated with $\lambda=\lambda_{1}$.

Proof. If $\Phi_{1}\left(x, t ; \lambda_{1}\right)$ is the solution of (5),

$$
\begin{aligned}
\phi_{110 x}\left(x, t ; \lambda_{1}\right)= & -i \lambda_{1} \phi_{110}\left(x, t ; \lambda_{1}\right) \\
& +q(x, t) \phi_{120}\left(x, t ; \lambda_{1}\right), \\
\phi_{111 x}\left(x, t ; \lambda_{1}\right)= & -i \lambda_{1} \phi_{111}\left(x, t ; \lambda_{1}\right) \\
& +q(x, t) \phi_{121}\left(x, t ; \lambda_{1}\right) \\
& +r(x, t) \phi_{120}\left(x, t ; \lambda_{1}\right), \\
\phi_{120 x}\left(x, t ; \lambda_{1}\right)= & i \lambda_{1} \phi_{120}\left(x, t ; \lambda_{1}\right) \\
& +q^{*}(-x, t) \phi_{110}\left(x, t ; \lambda_{1}\right), \\
\phi_{121 x}\left(x, t ; \lambda_{1}\right)= & i \lambda_{1} \phi_{121}\left(x, t ; \lambda_{1}\right) \\
& +q^{*}(-x, t) \phi_{111}\left(x, t ; \lambda_{1}\right) \\
& +r^{*}(-x, t) \phi_{110}\left(x, t ; \lambda_{1}\right) .
\end{aligned}
$$

Take the conjugate for the above equation on both sides at the same time, and let $x=-x$ :

$$
\begin{aligned}
\phi_{110 x}^{*}\left(-x, t ; \lambda_{1}\right)= & i \lambda_{1}^{*} \phi_{110}^{*}\left(-x, t ; \lambda_{1}\right) \\
& +q(-x, t)^{*} \phi_{120}^{*}\left(-x, t ; \lambda_{1}\right), \\
\phi_{111 x}^{*}\left(-x, t ; \lambda_{1}\right)= & i \lambda_{1}^{*} \phi_{111}^{*}\left(-x, t ; \lambda_{1}\right) \\
& +q(-x, t)^{*} \phi_{121}^{*}\left(-x, t ; \lambda_{1}\right) \\
& +r(-x, t)^{*} \phi_{120}^{*}\left(-x, t ; \lambda_{1}\right), \\
\phi_{120 x}^{*}\left(-x, t ; \lambda_{1}\right)= & -i \lambda_{1}^{*} \phi_{120}^{*}\left(-x, t ; \lambda_{1}\right) \\
& +q(x, t) \phi_{110}^{*}\left(-x, t ; \lambda_{1}\right), \\
\phi_{121 x}^{*}\left(-x, t ; \lambda_{1}\right)= & -i \lambda_{1}^{*} \phi_{121}^{*}\left(-x, t ; \lambda_{1}\right) \\
& +q(x, t) \phi_{111}^{*}\left(-x, t ; \lambda_{1}\right) \\
& +r(x, t) \phi_{110}^{*}\left(-x, t ; \lambda_{1}\right),
\end{aligned}
$$

so if $\lambda_{1}=-\lambda_{1}^{*}$, then

$$
\begin{aligned}
\phi_{110 x}^{*}\left(-x, t ;-\lambda_{1}^{*}\right)= & -i \lambda_{1} \phi_{110}^{*}\left(-x, t ;-\lambda_{1}^{*}\right) \\
& +q(-x, t)^{*} \phi_{120}^{*}\left(-x, t ;-\lambda_{1}^{*}\right), \\
\phi_{111 x}^{*}\left(-x, t ;-\lambda_{1}^{*}\right)= & -i \lambda_{1} \phi_{111}^{*}\left(-x, t ;-\lambda_{1}^{*}\right) \\
& +q(-x, t)^{*} \phi_{121}^{*}\left(-x, t ;-\lambda_{1}^{*}\right) \\
& +r(-x, t)^{*} \phi_{120}^{*}\left(-x, t ;-\lambda_{1}^{*}\right), \\
\phi_{120 x}^{*}\left(-x, t ;-\lambda_{1}^{*}\right)= & i \lambda_{1} \phi_{120}^{*}\left(-x, t ;-\lambda_{1}^{*}\right) \\
& +q(x, t) \phi_{110}^{*}\left(-x, t ;-\lambda_{1}^{*}\right), \\
\phi_{121 x}^{*}\left(-x, t ;-\lambda_{1}^{*}\right)= & i \lambda_{1} \phi_{121}^{*}\left(-x, t ;-\lambda_{1}^{*}\right) \\
& +q(x, t) \phi_{111}^{*}\left(-x, t ;-\lambda_{1}^{*}\right) \\
& +r(x, t) \phi_{110}^{*}\left(-x, t ;-\lambda_{1}^{*}\right) .
\end{aligned}
$$

Taking a similar procedure, the symmetry property also holds for the t-part of the Lax pair; that is, if $\lambda_{1}=-\lambda_{1}^{*}$, the eigenfunction

$$
\left(\begin{array}{c}
\phi_{120}^{*}\left(-x, t ;-\lambda_{1}^{*}\right)^{*} E+\phi_{121}^{*}\left(-x, t ;-\lambda_{1}^{*}\right) \Gamma \\
-\phi_{110}^{*}\left(-x, t ;-\lambda_{1}^{*}\right) E-\phi_{111}^{*}\left(-x, t ;-\lambda_{1}^{*}\right) \Gamma
\end{array}\right)
$$

is also the eigenfunction corresponding to $\lambda_{1}$, because it satisfies the same Lax equations. We can let

$$
\begin{aligned}
& \phi_{210}\left(x, t ; \lambda_{1}\right)=\phi_{120}^{*}\left(-x, t ;-\lambda_{1}^{*}\right), \\
& \phi_{211}\left(x, t ; \lambda_{1}\right)=\phi_{121}^{*}\left(-x, t ;-\lambda_{1}^{*}\right), \\
& \phi_{220}\left(x, t ; \lambda_{1}\right)=-\phi_{110}^{*}\left(-x, t ;-\lambda_{1}^{*}\right), \\
& \phi_{221}\left(x, t ; \lambda_{1}\right)=-\phi_{111}^{*}\left(-x, t ;-\lambda_{1}^{*}\right),
\end{aligned}
$$

and $\lambda_{2}=-\lambda_{1}^{*}$ in the following. 
According to Lemma 1 , by choosing $a_{10}=a_{11}=1, d_{10}=$ $d_{11}=1$, we can get the onefold Darboux transformation for the weakly coupled NLS equation as follows:

$$
\begin{aligned}
& T=T_{1}(\lambda) \\
& =\left(\begin{array}{cc}
\left(\lambda+a_{0}\right) E+\left(\lambda+a_{1}\right) \Gamma & b_{0} E+b_{1} \Gamma \\
c_{0} E+c_{1} \Gamma & \left(\lambda+d_{0}\right) E+\left(\lambda+d_{1}\right) \Gamma
\end{array}\right) \\
& =\left(\begin{array}{cc}
E+\Gamma & 0 \\
0 & E+\Gamma
\end{array}\right) \lambda+\left(\begin{array}{cc}
a_{0} E+a_{1} \Gamma & b_{0} E+b_{1} \Gamma \\
c_{0} E+c_{1} \Gamma & d_{0} E+d_{1} \Gamma
\end{array}\right),
\end{aligned}
$$

and then the new solution $q^{[1]}, r^{[1]}$ can be obtained by the Darboux transformation

$$
\begin{aligned}
& q^{[1]}(x, t)=q(x, t)+2 i b_{0}, \\
& r^{[1]}(x, t)=r(x, t)+2 i\left(b_{1}-b_{0}\right) .
\end{aligned}
$$

The Darboux transformation matrix $T_{1}$ must satisfy the following equation:

$$
T_{1}\left(\lambda, \lambda_{j}\right) \Phi_{j}=0, \quad j=1,2
$$

From the above equation, we can get the expressions of functions $b_{0}, b_{1}$ easily by the eigenfunctions as follows:

$$
\begin{aligned}
& b_{0}=\frac{\left(\lambda_{1}-\lambda_{2}\right) \phi_{110} \phi_{210}}{\phi_{110} \phi_{220}-\phi_{120} \phi_{210}} \\
& b_{1}=\frac{\left(\lambda_{1}-\lambda_{2}\right)\left(\phi_{110}^{2}\left(\phi_{211} \phi_{220}-\phi_{210} \phi_{221}\right)+\phi_{210}^{2}\left(\phi_{110} \phi_{121}-\phi_{111} \phi_{120}\right)\right)}{\left(\phi_{110} \phi_{220}-\phi_{120} \phi_{210}\right)^{2}} .
\end{aligned}
$$

Combining with (28), the following theorem can be derived.

$$
\begin{aligned}
& q^{[1]}=q+2 i \frac{\left(\lambda_{1}-\lambda_{2}\right) \phi_{110} \phi_{210}}{\phi_{110} \phi_{220}-\phi_{120} \phi_{210}} \\
& r^{[1]}=r+2 i \frac{\left(\lambda_{1}-\lambda_{2}\right)\left(\phi_{110}^{2}\left(\phi_{220}\left(\phi_{211}-\phi_{210}\right)-\phi_{210} \phi_{221}\right)+\phi_{210}^{2}\left(\phi_{110}\left(\phi_{120}+\phi_{121}\right)-\phi_{111} \phi_{120}\right)\right)}{\left(\phi_{110} \phi_{220}-\phi_{120} \phi_{210}\right)^{2}}
\end{aligned}
$$

Then, degenerated Darboux transformation of the weakly coupled NLS equation is given explicitly by a series expansion of the $\phi_{i j k}\left(\lambda=\lambda_{1}+\epsilon\right), i, j=1,2, k=0,1$. Degenerated Darboux transformation of the weakly coupled nonlocal NLS equation will help us to find the rational solution in the next section.

\section{The Rational Solutions of the Weakly Coupled Nonlocal NLS Equation}

From the previous section, degenerated Darboux transformation of the weakly coupled nonlocal NLS equation has been discussed. In this section, we will be focused on a kind of solutions which starts from seed solutions by the degenerated Darboux transformation. The solutions are called rational solutions. The seed solutions can be assumed as $(q(x, t), r(x, t))=\left(c e^{i b t}, i c e^{i b t}\right), b, c$ being arbitrary real constants. Substituting seed solution into (4), through a proper simplification, we can obtain a constraint

$$
b=-2 c^{2}
$$

Theorem 2. The Darboux transformation of the weakly coupled NLS equation can be written:
Taking the transformation

$$
\begin{aligned}
\Phi_{x}(x, t ; \lambda) & =\psi_{x}(x, t ; \lambda) e^{i b t \sigma}, \\
\sigma & =\left(\begin{array}{cc}
1 & 0 \\
0 & -1
\end{array}\right),
\end{aligned}
$$

then

$$
\begin{aligned}
& \psi_{x}(x, t ; \lambda)=\widehat{M} \psi(x, t ; \lambda)=\left(\begin{array}{cc}
-i \lambda E & c E \\
c E & i \lambda E
\end{array}\right), \\
& \psi_{t}(x, t ; \lambda)=\widehat{N} \psi(x, t ; \lambda)=\left(\begin{array}{cc}
-2 i \lambda^{2} E & 2 c \lambda E \\
2 c \lambda E & 2 i \lambda^{2} E
\end{array}\right) .
\end{aligned}
$$

So solving the Lax pair equation (34) is equivalent to solving (5).

By defining

$$
R=\sqrt{c^{2}-\lambda^{2}}
$$


the following basic solutions $\psi_{110}, \psi_{111}, \psi_{120}, \psi_{121}$ are obtained in terms of $R$ from the the spectral problem of (5) as

$$
\begin{aligned}
& \psi_{110}(R)=e^{2 t R \lambda+x R}, \\
& \psi_{111}(R)=e^{2 t R \lambda+x R}, \\
& \psi_{120}(R)=\frac{(R+i \lambda)}{c} e^{2 t R \lambda+x R}, \\
& \psi_{121}(R)=\frac{(R+i \lambda)}{c} e^{2 t R \lambda+x R .}
\end{aligned}
$$

Here Lemma 1 and the superposition principle are adopted to construct the nontrivial eigenfunctions $\phi_{110}, \phi_{120}$, $\phi_{111}, \phi_{121}$ :

$$
\begin{aligned}
\phi_{110} & =J \psi_{110}(R)(x, t, \lambda)+K \psi_{120}^{*}(R)(-x, t,-\lambda) \\
& =J e^{2 t R \lambda+x R}+K \frac{(R+i \lambda)}{c} e^{-2 t R \lambda-x R}, \\
\phi_{111} & =J \psi_{111}(R)(x, t, \lambda)+K \psi_{121}^{*}(R)(-x, t,-\lambda) \\
& =J e^{2 t R \lambda+x R}+K \frac{(R+i \lambda)}{c} e^{-2 t R \lambda-x R,} \\
\phi_{120} & =J \psi_{120}(R)(x, t, \lambda)-K \psi_{110}^{*}(R)(-x, t,-\lambda) \\
& =J \frac{(R+i \lambda)}{c} e^{2 t R \lambda+x R}-K e^{-2 t R \lambda-x R}, \\
\phi_{121} & =J \psi_{121}(R)(x, t, \lambda)-K \psi_{111}^{*}(R)(-x, t,-\lambda) \\
& =J \frac{(R+i \lambda)}{c} e^{2 t R \lambda+x R}-K e^{-2 t R \lambda-x R,}
\end{aligned}
$$

where $J=J_{1}+i J_{2}, K=K_{1}+i K_{2}, J_{1}, J_{2}, K_{1}, K_{2}$ are arbitrary real constants. It can be proved that $\phi_{110}, \phi_{111}, \phi_{120}$, and $\phi_{121}$ are also the solutions of the Lax equation with $\lambda_{1}=c+\epsilon$. Now applying the degenerated Darboux transformation, we can get one kind of rational solution of the nonlocal NLS equation at the threshold $\lambda=c$ :

$$
\begin{aligned}
q^{[1]} & =\frac{F_{1}}{G_{1}} e^{-2 i t}, \\
r^{[1]} & =\frac{F_{2}+F_{3}+F_{4}}{G_{1}^{2}} e^{-2 i t},
\end{aligned}
$$

where

$$
\begin{aligned}
F_{1} & =-8\left(J_{1}^{2}+J_{2}^{2}-2 J_{2}+1\right) t^{2}-8\left(i J_{1}^{2}+i J_{2}^{2}-2 i J_{2}+i\right. \\
& \left.+J_{1}\right) t-2\left(2 J_{2}-J_{1}^{2}-J_{2}^{2}-1\right) x^{2}-2\left(i J_{2}^{2}+i J_{1}^{2}-i\right) \\
& \cdot x-4 i J_{1}+J_{1}^{2}+J_{2}^{2}-4 J_{2}+1, \\
G_{1} & =8\left(J_{1}^{2}+J_{2}^{2}-2 J_{2}+1\right) t^{2}+8 J_{1} t+2\left(2 J_{2}-J_{1}^{2}-J_{2}^{2}\right. \\
& -1) x^{2}+2 i\left(J_{1}^{2}+J_{2}^{2}-1\right) x+J_{1}^{2}+J_{2}^{2}+1,
\end{aligned}
$$

$$
\begin{aligned}
& F_{2}=((2+i) \\
& \cdot\left(64\left(J_{1}^{2}+J_{2}^{2}\right)^{2}-256 J_{2}\left(J_{2}^{2}+J_{1}^{2}+1\right)+J_{2}^{2}\right) \\
& \left.+128 i J_{1}^{2}+64(6+i)\right) t^{4}+64\left(i\left(J_{2}^{2}+J_{1}^{2}\right)^{2}\right. \\
& +4\left(J_{2}-1\right)^{2}\left(J_{1}-i J_{2}\right) \\
& +2 J_{1}\left(i\left(\left(J_{1}+1\right)^{2}-J_{1}\left(1+2 J_{2}\right)\right)+2\left(J_{1}^{2}-i J_{2}\right)\right) \\
& \left.+2 i J_{2}^{2}\left(J_{1}-1\right)+i\right) t^{3}+(16(i+1) \\
& \cdot\left(\left(J_{1}^{2}+J_{2}^{2}\right)^{2}+1\right)+32 J_{1}^{2}\left(3 i\left(1+J_{1}\right)-i J_{2}+5\right) \\
& +32 J_{2}^{2}\left(3 i J_{1}-i J_{2}+i-1\right)-32 i J_{2} \\
& \left.+96 i J_{1}\left(1-2 J_{2}\right)\right) t^{2} \\
& +8\left(2 i J_{2}\left(J_{2}-1-\left(\left(J_{1}^{2}-J_{2}\right)^{2}\right)\right)\right. \\
& +J_{1}\left(6 i J_{1}+2(i+1)+J_{1}^{2}\left(2(1+i)-i J_{1}\right)\right) \\
& \left.+i\left(\left(J_{1}^{2}+J_{2}^{2}\right)^{2}+J_{1}^{4}+1\right)\right) t, \\
& F_{3}=4(2+i)\left(\left(J_{1}^{2}+J_{2}^{2}\right)^{2}-\left(4 J_{2}^{2}-6 J_{2}+8\right) J_{2}\right. \\
& \left.+2 J_{1}^{2}\left(1-2 J_{2}\right)+1\right) x^{4}+(2 i-1)\left(8-8\left(J_{1}^{2}+J_{2}^{2}\right)^{2}\right. \\
& \left.+J_{2}\left(J_{1}^{2}+16\left(J_{2}^{2}-1\right)\right)\right) x^{3} \\
& +\left(J_{1}^{2}\left(8\left(1+i J_{2}-i J_{1}\right)-12 J_{1}^{2}\right)\right) \\
& -8 i\left(J_{2}^{2}\left(24-12 J_{2}^{2}+8 i J_{2}\right)-12+\left(J_{1}^{2}+J_{2}^{2}\right)^{2}\right. \\
& \left.+J_{1}\left(1-J_{2}\right)^{2}\right) x^{2}+\left(4(i-1)\left(\left(J_{1}^{2}+J_{2}^{2}\right)^{2}-1\right)\right. \\
& \left.+8\left(J_{1}\left(J_{2}\left(i J_{1}-J_{2}\right)+1\right)-J_{1}^{3}-i J_{2}+i J_{2}^{3}\right)\right) x, \\
& F_{4}=\left(J_{1}^{2}\left(64(2+i)\left(2 J_{2}-1\right)-24 J_{2}^{2}\right)+(2+i)\right. \\
& \left.\cdot\left(128 J_{2}-32\left(J_{1}^{2}+J_{2}^{2}\right)^{2}+J_{2}^{3}-192 J_{2}^{2}-32\right)\right) t^{2} x^{2} \\
& +32\left((2 i-1)\left(J_{1}^{2}+J_{2}^{2}\right)^{2}+J_{1}^{2}\left(1+2 J_{2}(1-2 i)\right)\right. \\
& \left.-2 J_{2}(1-2 i)\left(1-J_{2}^{2}\right)-2 i\right) t^{2} x \\
& +\left(32 J_{2}^{2}\left(2 i J_{2}-2 i-J_{1}(2+i)\right)-32 i\left(J_{1}-J_{2}\right)^{2}\right. \\
& -16 i\left(J_{1}^{2}+J_{2}^{2}\right)^{2}-16 i-32 J_{1}^{3}(i+2) \\
& \left.+32 J_{1}\left(4 J_{2}-i-2\right)+64 i J_{2}\left(J_{1}^{2}+1\right)\right) t x^{2}+32\left(J_{2}^{3}\right. \\
& +J_{2}\left(J_{1}^{2}-1\right)-16\left(J_{1}^{2}+J_{2}^{2}\right)^{2}+(2 i-1)
\end{aligned}
$$




$$
\begin{aligned}
& \left.\cdot J_{1}\left(J_{1}^{2}+J_{2}^{2}-1\right)\right) t x+i+2 i J_{1}^{2}\left(2 J_{2}+1\right)+2 i J_{2}^{2}(1 \\
& \left.+2 J_{1}\right)+i\left(J_{1}^{2}+J_{2}^{2}\right)^{2}+4 i\left(J_{1}+J_{1}^{3}\right)+4\left(J_{2}+4 J_{2}^{3}\right) .
\end{aligned}
$$

Here, for convenience, we have set $c=1$. It can be verified that the solutions (38) satisfy the PT-symmetry. Furthermore, the solutions are analytic under certain condition.

Theorem 3. If $J_{1}^{2}+J_{2}^{2} \neq 1$, then $\left|q^{[1]}\right|$ and $\left|r^{[1]}\right|$ are analytic. Otherwise, $\left|q^{[1]}\right|$ and $\left|r^{[1]}\right|$ have singular points.

Proof. First, we display that $\left|q^{[1]}\right|$ and $\left|r^{[1]}\right|$ are analytic when $J_{1}^{2}+J_{2}^{2} \neq 1$. In (38) $\left|q^{[1]}\right|$ and $\left|r^{[1]}\right|$ are rational solutions, so the singular points just come from zero points of the denominator $\left|G_{1}\right|$; that is, it is enough to prove $\left|G_{1}\right| \neq 0$ when $J_{1}^{2}+J_{2}^{2} \neq 1$.

We know that $\left|G_{1}\right|=0$ is equivalent to $G_{1 R}=0$ and $G_{1 I}=$ 0 , where $G_{1 R}$ and $G_{1 I}$ denote the real part and imaginary part of $G_{1}$, respectively.
If $G_{1 I}=0$, we can get $x=0$ in the case of $J_{1}^{2}+J_{2}^{2} \neq 1$. Substituting $x=0$ into $G_{1 R}=0$, then

$$
\begin{aligned}
G_{1 R} & =\left(8 J_{1}^{2}+8 J_{2}^{2}-16 J_{2}+8\right) t^{2}+8 J_{1} t+J_{1}^{2}+J_{2}^{2}+1 \\
& =0,
\end{aligned}
$$

which implies

$$
\begin{aligned}
t & =\frac{-2 J_{1} \pm \sqrt{\Delta_{1}}}{4\left(J_{1}^{2}+J_{2}^{2}-2 J_{2}+1\right)} \\
\Delta_{1} & =-2\left(J_{1}^{2}+J_{2}^{2}-J_{2}\right)^{2}-2\left(J_{2}-1\right)^{2} .
\end{aligned}
$$

Through simple calculation, we find that $\Delta_{1}$ arrives at its maximum value 0 when $J_{1}=0$ and $J_{1}=1$. Since $J_{1}^{2}+J_{2}^{2} \neq$ 1 is always negative, this implies there is no $t \in R$ making $G_{1 R}=0$. Therefore, the rational solutions (38) are analytic when $J_{1}^{2}+J_{2}^{2} \neq 1$.

Second, let $J_{1}^{2}+J_{2}^{2}=1$ in (38). For convenience, we define $J_{1}=\cos (\theta)$ and $J_{2}=\sin (\theta)$. Then we can get

$$
\begin{aligned}
& q^{[1]}=\frac{-8 i t \sin (\theta)-8 t^{2} \sin (\theta)+2 x^{2} \sin (\theta)+2 i \cos (\theta)+8 i t+4 t \cos (\theta)+8 t^{2}-2 x^{2}+2 \sin (\theta)-1}{Q} \\
& r^{[1]}=\frac{\left(i\left(\sin (\theta)\left(-16 i t^{2}+4 i x^{2}+8 t^{2}-2 x^{2}+2 i+8 t\right)+\cos (\theta)(8 i t-2-4 t)-8 t^{2}+2 x^{2}+16 i t^{2}-4 i x^{2}-8 t-1\right)\right)}{Q},
\end{aligned}
$$

where $Q=(2-2 \sin (\theta)) x^{2}+8 \sin (\theta) t^{2}-4 t \cos (\theta)-8 t^{2}-1$.

When $\sin (\theta)=1,\left(q^{[1]}, r^{[1]}\right)$ are analytic but trivial owing to $q^{[1]}=-1, r^{[1]}=2+I$ in this case. So when $\sin (\theta) \neq 1$ we need to prove that there always exist $x \in R$ and $t \in R$ making $Q$ be zero.

Now we need to prove the discriminant of $Q$ about $x$

$$
\begin{aligned}
& \Delta_{2} \\
& \quad=8(\sin (\theta)-1)\left(8 \sin (\theta) t^{2}-4 \cos (\theta) t-8 t^{2}-1\right)
\end{aligned}
$$

is positive. Owing to $\sin (\theta)-1<0$, let $f(\theta)=8 t^{2}(\sin (\theta)-$ $1)-4 \cos (\theta) t-1$; we need to prove $f(\theta)<0$. Let $f\left(\theta_{0}\right)^{\prime}=0$; then $\theta_{0}=-\arctan (2 t)$, taking $\theta_{0}$ into $f(\theta)$, leading to $f\left(\theta_{0}\right)=$ $-4 t \sqrt{4 t^{2}+1}-8 t^{2}-1$. Obviously, there exists $t \in R$ making $f\left(\theta_{0}\right)<0$ so that the discriminant $\Delta_{2}>0$. Thus, $q^{[1]}$ and $r^{[1]}$ have singular points when $J_{1}^{2}+J_{2}^{2}=1$.

The dynamical structures of $\left(\left|q_{1}\right|^{2},\left|r_{1}\right|^{2}\right)$ under the condition $J_{1}^{2}+J_{2}^{2} \neq 1$ were shown in Figures 1 and 3 . When $x \longrightarrow \infty, t \longrightarrow \infty$, we can know $\left|q^{[1]}\right|^{2} \longrightarrow 1,\left|r^{[1]}\right|^{2} \longrightarrow 5$ with $c=1$. In Figure 1, we find that $\left(\left|q_{1}\right|^{2}\right)$ has four types of patterns. The first and second cases, shown in Figures 1(a) and 1(b), display two wavefronts, a bright one and a dark one, moving from $t=-\infty$ to $t=\infty$ on two straight lines and intersecting with each other in the neighborhood of the origin. At the intersection point, amplitudes of the bright and the dark wavelet decrease rapidly, and then their amplitudes simultaneously increase rapidly to their original amplitudes before the intersection. The only difference between Figures 1(a) and 1(b) is that the bright and dark wavefronts exchange their locations. The third and fourth cases, shown in Figures 1(c) and 1(d), display the evolution of two bright wavefronts, and the amplitudes of these bright wavefronts have similar properties as the wavefronts in the first and second cases. In Figure 3, we find that $\left(\left|r_{1}\right|^{2}\right)$ has two types of patterns. The first case, shown in Figure 3(a), is similar to Figure 1(b). The second case, shown in Figure 3(b), is similar to Figure 1(a). Figure 2 and Figure 4 are density plots of Figure 1 and Figure 3, respectively.

In the end of the section, we study the self-induced potential function $V^{[1]}(x, t)$, the PT-symmetric potential $V^{[1]}(x, t)=V_{R}^{[1]}+\mathrm{i} V_{I}^{[1]}=q^{[1]}(x, t) q^{[1]}(-x, t)$,

$$
\begin{aligned}
V^{[1]} & (x, t)=-\frac{F_{5}}{F_{6}} \\
F_{5} & =\left(8 i t J_{1}^{2}+2 i x J_{1}^{2}+8 i t-2 i x+8 t^{2} J_{1}^{2}+8 t^{2} J_{2}^{2}\right. \\
& -2 x^{2} J_{1}^{2}-2 x^{2} J_{2}^{2}-16 i t J_{2}-16 t^{2} J_{2}+4 x^{2} J_{2}+4 i J_{1} \\
& +8 i t J_{2}^{2}+2 i x J_{2}^{2}+8 t^{2}+8 t J_{1}-2 x^{2}-J_{1}^{2}-J_{2}^{2}+4 J_{2} \\
& -1)\left(1-8 t^{2}-4 J_{2}+8 i t J_{1}^{2}+8 i t-2 i x J_{1}^{2}+2 i x+J_{1}^{2}\right. \\
& +J_{2}^{2}-(16 i) t J_{2}-8 t^{2} J_{1}^{2}-8 t^{2} J_{2}^{2}+16 t^{2} J_{2}+2 x^{2} J_{1}^{2}
\end{aligned}
$$




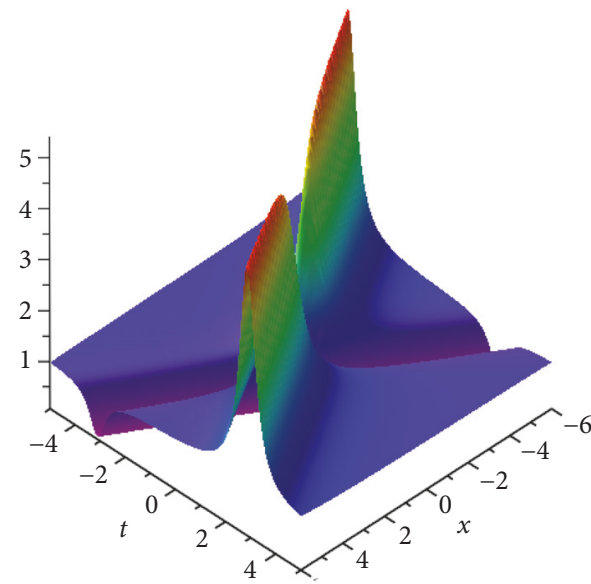

(a) $\left(\left|q^{[1]}\right|^{2}\right)$

(c) $\left(\left|q^{[1]}\right|^{2}\right)$

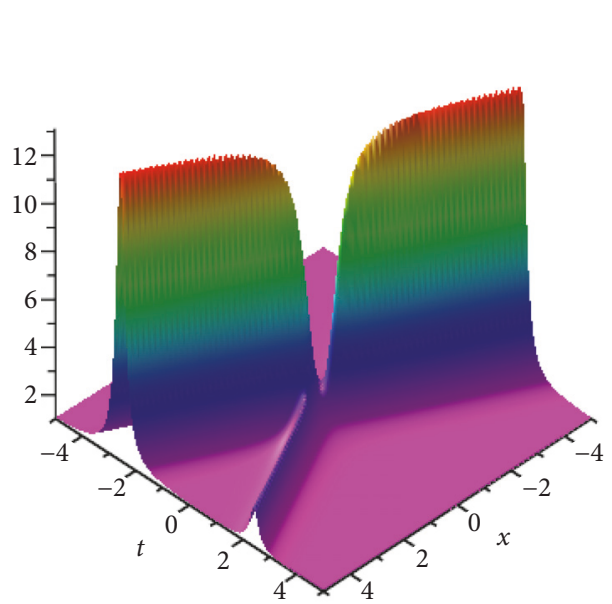

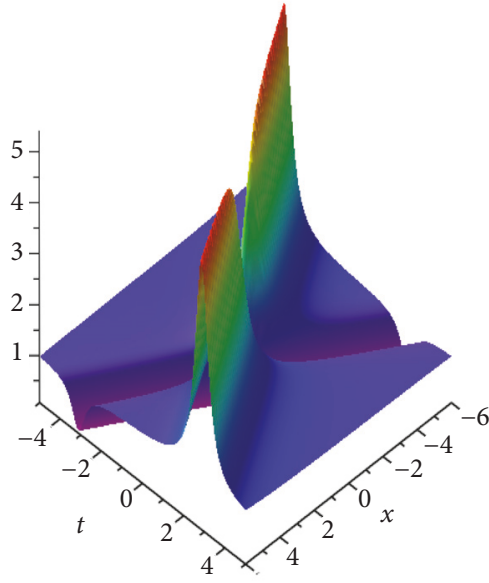

(b) $\left(\left|q^{[1]}\right|^{2}\right)$

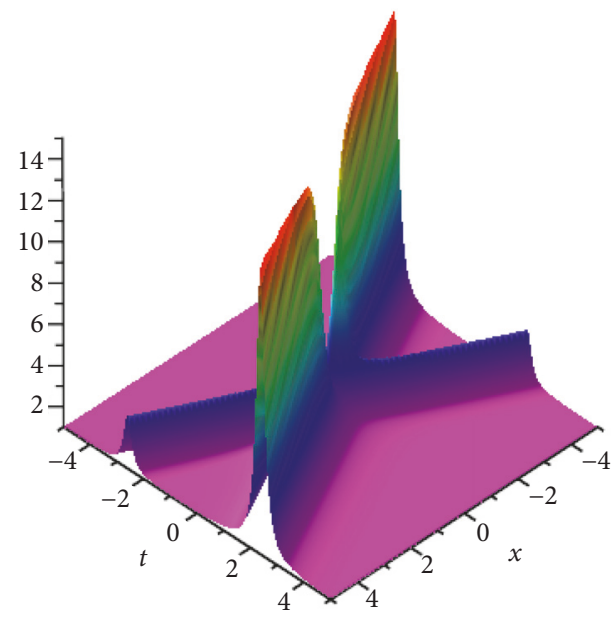

(d) $\left(\left|q^{[1]}\right|^{2}\right)$

FIGURE 1: $\left|q^{[1]}\right|^{2}$ displays four structures with the height of background being 1. (a) The dark-bright structure: $\left(J_{1}, J_{2}\right)=(2,3)$. (b) The brightdark structure: $\left(J_{1}, J_{2}\right)=(0.2,0.2)$. (c) The bright-bright 1 structure: $\left(J_{1}, J_{2}\right)=(0.2,-0.1)$. (d) The bright-bright 2 structure: $\left(J_{1}, J_{2}\right)=(3,-1)$.

$$
\begin{aligned}
& +2 x^{2} J_{2}^{2}-4 x^{2} J_{2}-8 t J_{1}+2 x^{2}+4 i J_{1}+8 i t J_{2}^{2} \\
& \left.-2 i x J_{2}^{2}\right), \\
F_{6} & =\left(8 t^{2} J_{1}^{2}+8 t^{2} J_{2}^{2}-16 t^{2} J_{2}+8 t^{2}-2 x^{2} J_{1}^{2}-2 x^{2} J_{2}^{2}\right. \\
& +2 i x J_{1}^{2}+2 i x J_{2}^{2}+4 x^{2} J_{2}+8 t J_{1}-2 x^{2}-2 i x+J_{1}^{2} \\
& \left.+J_{2}^{2}+1\right)^{2} .
\end{aligned}
$$

Figures 5 and 6 imply that in the PT-symmetric waveguide system that involved refractive index and gain or loss profiles, when a part of the system involves loss, an equal amount of gain is observed near this part, which may be applied to overcome the loss effects in the system. Thus, our results may guide the interplay between gain and loss and the manufacture of a new generation of multifunction optical devices and networks.

\section{Conclusion}

In this article, we study a weakly coupled nonlocal NLS equation. We have shown its integrability by proposing its Lax pair. Then, we have derived the Darboux transformation of the weakly coupled nonlocal NLS equation which implies the representations of $\left(q^{[1]}, r^{[1]}\right)$ generated from known solutions $(q, r)$. By choosing some special eigenvalues $\lambda_{2}=-\lambda_{1}^{*}$, and eigenfunctions using the reduction conditions $\varphi_{210}\left(x, t, \lambda_{2}\right)=\varphi_{120}^{*}\left(-x, t,-\lambda_{1}^{*}\right), \varphi_{211}\left(x, t, \lambda_{2}\right)=$ $\varphi_{121}^{*}\left(-x, t,-\lambda_{1}^{*}\right), \quad \varphi_{220}\left(x, t, \lambda_{2}\right)=-\varphi_{110}^{*}\left(-x, t,-\lambda_{1}^{*}\right)$, $\varphi_{221}\left(x, t, \lambda_{2}\right)=-\varphi_{111}^{*}\left(-x, t,-\lambda_{1}^{*}\right)$, the representations of 


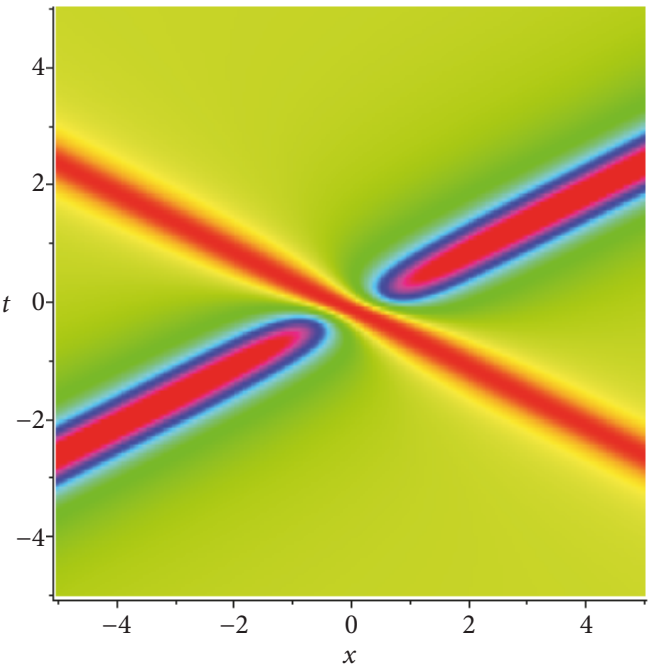

(a) $\left(\left|q^{[1]}\right|^{2}\right)$

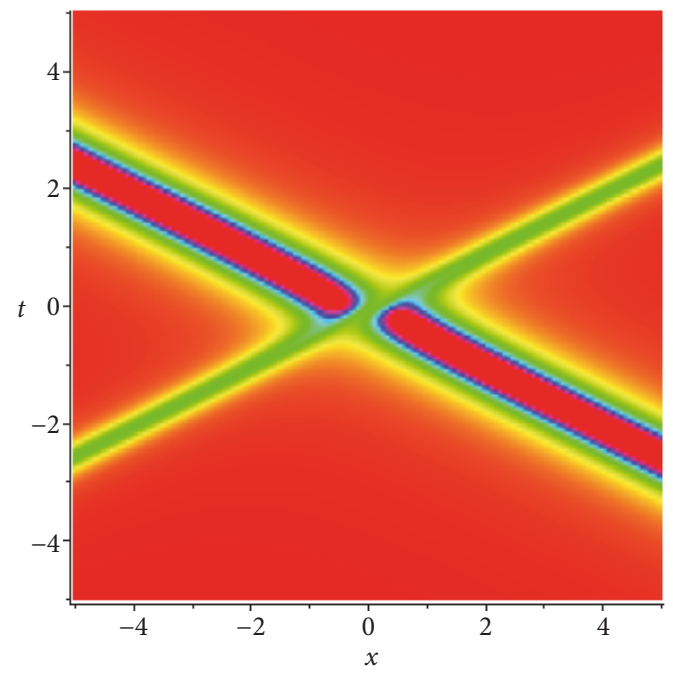

(c) $\left(\left|q^{[1]}\right|^{2}\right)$

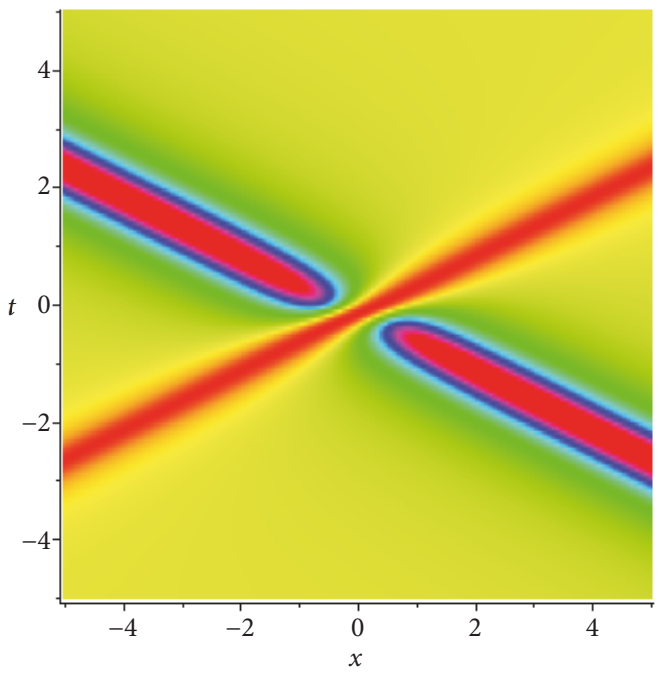

(b) $\left(\left|q^{[1]}\right|^{2}\right)$

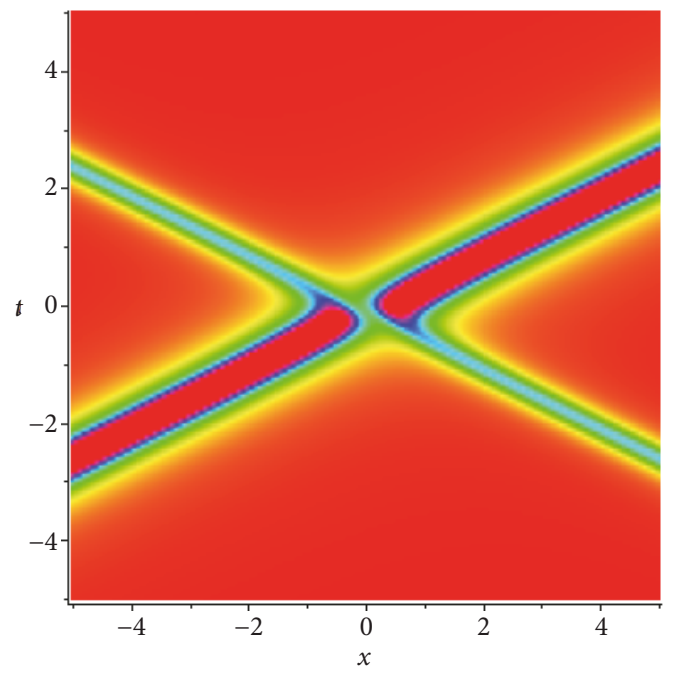

(d) $\left(\left|q^{[1]}\right|^{2}\right)$

Figure 2: Density plots of $\left|q^{[1]}\right|^{2}$ on $(x, t)$-plane associated with Figure 1. (a) The dark-bright structure: $\left(J_{1}, J_{2}\right)=(2,3)$. (b) The bright-dark structure: $\left(J_{1}, J_{2}\right)=(0.2,0.2)$. (c) The bright-bright 1 structure: $\left(J_{1}, J_{2}\right)=(0.2,-0.1)$. (d) The bright-bright 2 structure: $\left(J_{1}, J_{2}\right)=(3,-1)$.

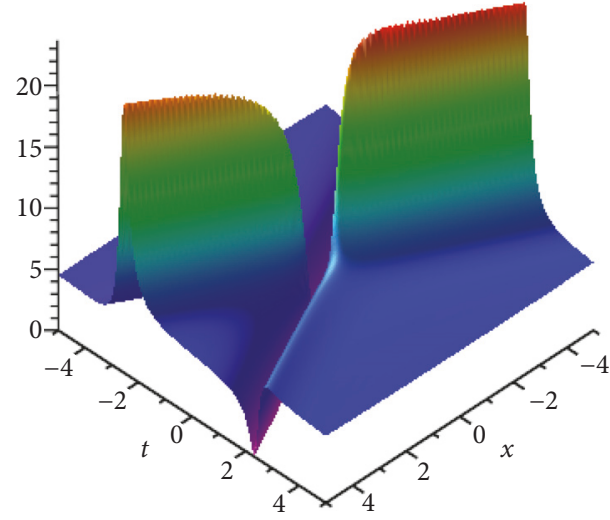

(a) $\left(\left|r^{[1]}\right|^{2}\right)$

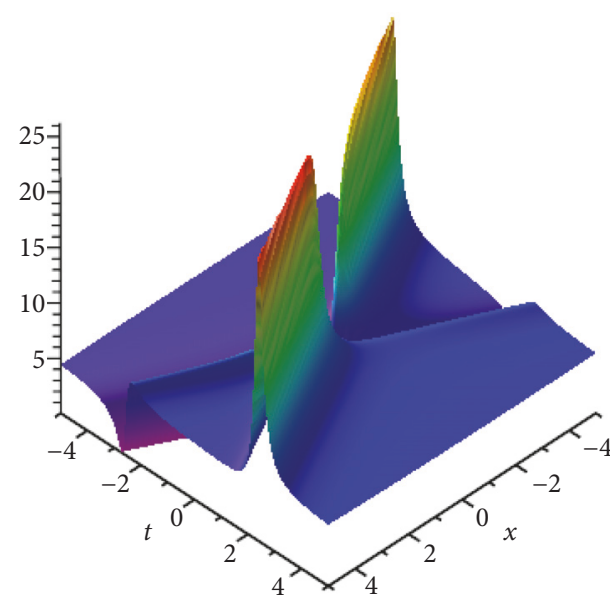

(b) $\left(\left|r^{[1]}\right|^{2}\right)$

FIGURE 3: $\left|r^{[1]}\right|^{2}$ displays two structures with the height of background being 5. (a) The bright-bright 1 structure: $\left(J_{1}, J_{2}\right)=(0.2,-0.1)$. (b) The bright-bright 2 structure: $\left(J_{1}, J_{2}\right)=(3,-1)$. 


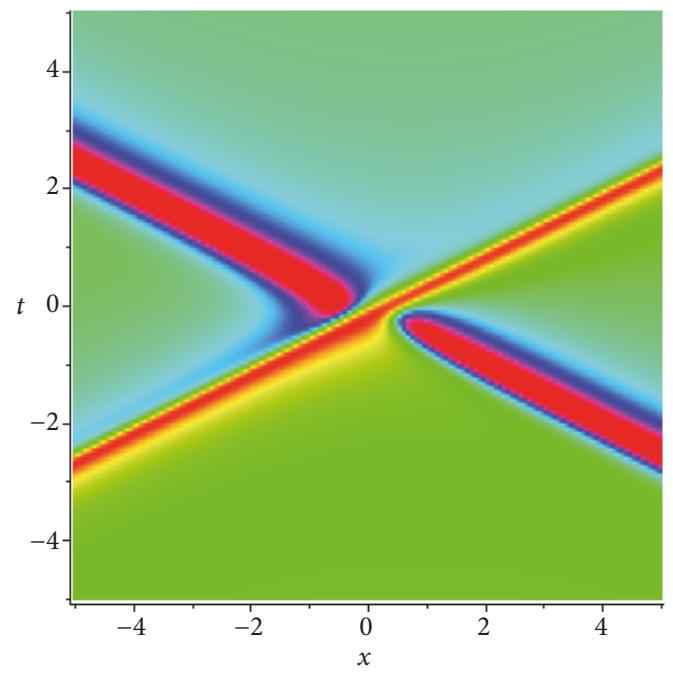

(a) $\left(\left|r^{[1]}\right|^{2}\right)$

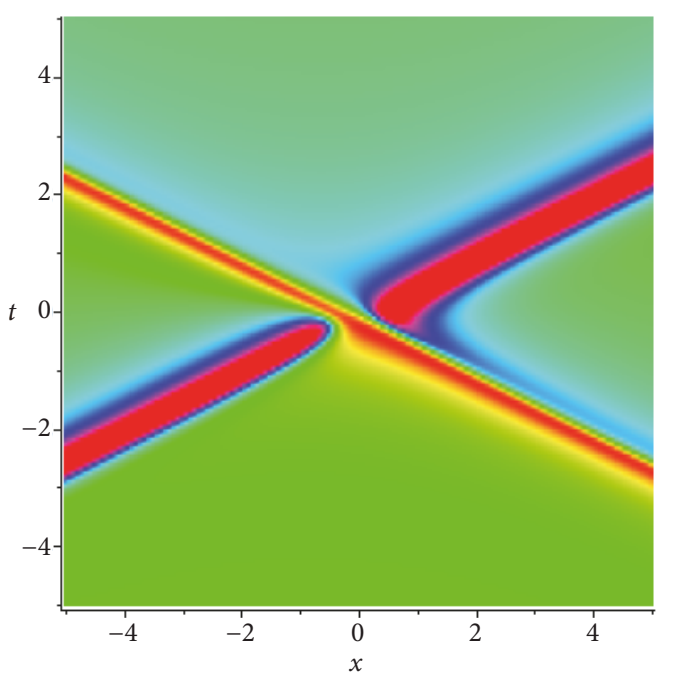

(b) $\left(\left|r^{[1]}\right|^{2}\right)$

Figure 4: Density plots of $\left|r^{[1]}\right|^{2}$ on $(x, t)$-plane associated with Figure 1 . (a) The bright-bright 1 structure: $\left(J_{1}, J_{2}\right)=(0.2,-0.1)$. (b) The bright-bright 2 structure: $\left(J_{1}, J_{2}\right)=(3,-1)$.
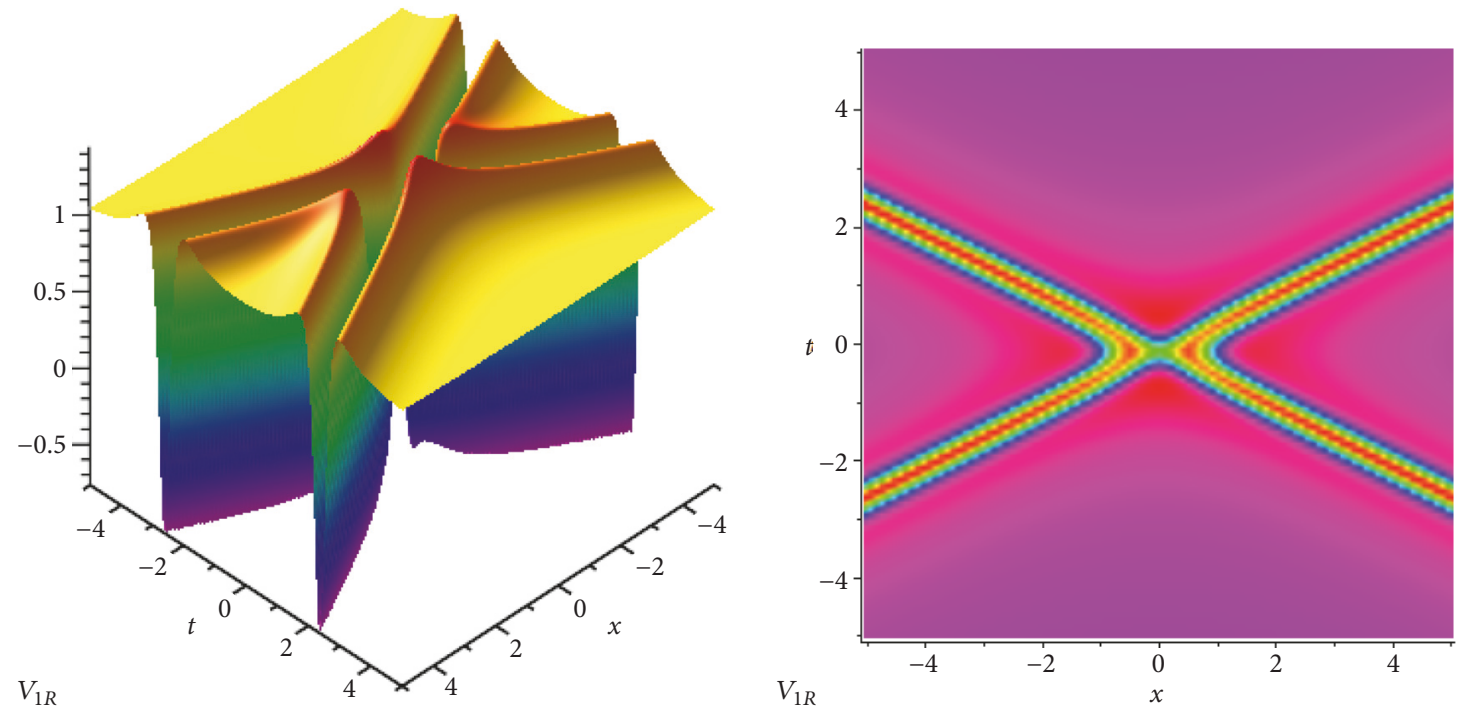

FIgURE 5: The real part of the PT-symmetric potential generated by the rational solution. The profile of $V_{1 I}$ with $\left(J_{1}, J_{2}\right)=(2,3)$.

(31) provided some different solutions of the weakly coupled nonlocal NLS equation. By using degenerate Darboux transformation, we have derived rational solutions, the real refractive index profile $\left(V_{R}^{[1]}\right)$, and the gain-or-loss distribution $\left(V_{I}^{[1]}\right)$ of the weakly coupled nonlocal NLS equation under the PT-symmetry condition and we have proved that it is analytic if $J_{1}^{2}+J_{2}^{2} \neq 1$ [17]. We have divided the obtained solutions $q^{[1]}, r^{[1]}$ into four categories solutions and two categories solutions which are graphically illustrated in Figures 1 and 3, respectively. This observation may be useful for synthesizing new artificial optical structures and materials by mixing together the refractive index distribution and the gain-or-loss profile.

\section{Data Availability}

There is no data in this manuscript.

\section{Conflicts of Interest}

The authors declare that there are no conflicts of interest regarding the publication of this paper. 

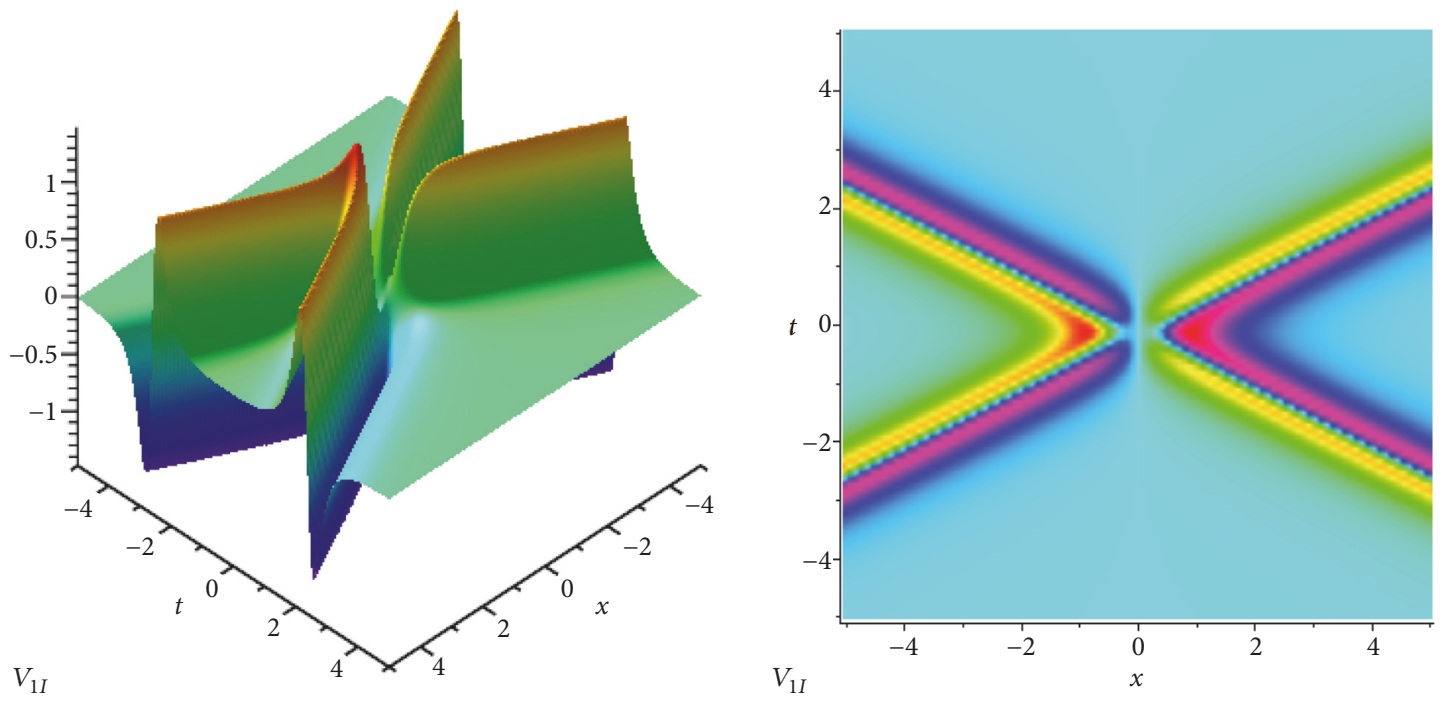

FIgURE 6: The imaginary part of the PT-symmetric potential generated by the rational solution. The profile of $V_{1 I}$ with $\left(J_{1}, J_{2}\right)=(2,3)$.

\section{Acknowledgments}

Chuanzhong Li is supported by the National Natural Science Foundation of China under Grant no. 11571192 and K. C. Wong Magna Fund in Ningbo University.

\section{References}

[1] B. Bagchi, S. Modak, and P. K. Panigrahi, “Tracking down localized modes in pt-symmetric hamiltonians under the influence of a competing nonlinearity," Acta Polytechnica, vol. 54, no. 2 , pp. 79-84, 2014.

[2] C. M. Bender and S. Boettcher, "Real spectra in non-Hermitian Hamiltonians having PT symmetry," Physical Review Letters, vol. 80, no. 24, pp. 5243-5246, 1998.

[3] B. Bagchi and C. Quesne, "Non-Hermitian HAMiltonians with real and complex eigenvalues in a Lie-algebraic framework," Physics Letters A, vol. 300, no. 1, pp. 18-26, 2002.

[4] C. M. Bender, D. C. Brody, H. F. Jones, and B. K. Meister, "Faster than Hermitian quantum mechanics," Physical Review Letters, vol. 98, no. 4, 040403, 4 pages, 2007.

[5] K. G. Makris, R. El-Ganainy, Z. H. Musslimani, and D. N. Christodoulides, "Theory of coupled optical PT-symmetric structures," Optics Expresss, vol. 32, no. 17, pp. 2632-2634, 2007.

[6] Z. H. Musslimani, K. G. Makris, R. El-Ganainy, and D. N. Christodoulides, "Optical Solitons in ," Physical Review Letters, vol. 100, no. 3, 2008.

[7] K. G. Makris, R. El-Ganainy, D. N. Christodoulides, and Z. H. Musslimani, "Beam dynamics in PT symmetric optical lattices," Physical Review Letters, vol. 100, no. 10, Article ID 103904, 2008.

[8] C. E. Rüter, K. G. Makris, R. El-Ganainy, D. N. Christodoulides, M. Segev, and D. Kip, "Observation of parity-time symmetry in optics," Nature Physics, vol. 6, no. 3, pp. 192-195, 2010.

[9] A. Regensburger, C. Bersch, M.-A. Miri, G. Onishchukov, D. N. Christodoulides, and U. Peschel, "Parity-time synthetic photonic lattices," Nature, vol. 488, no. 7410, pp. 167-171, 2012.

[10] A. Regensburger, M. Miri, C. Bersch et al., " Observation of Defect States in ," Physical Review Letters, vol. 110, no. 22, 2013.
[11] A. Guo, G. J. Salamo, D. Duchesne et al., "Observation of PT-symmetry breaking in complex optical potentials," Physical Review Letters, vol. 103, no. 9, Article ID 093902, 2009.

[12] V. E. Zakharov and A. B. Shabat, "Exact theory of twodimensional self-focusing and one-dimensional self-modulation of waves in nonlinear media," Sov. Phys. JETP, vol. 34, Article ID 6353506, 1972.

[13] D. J. Benney and A. C. Newell, "The propagation of nonlinear wave envelopes," Studies in Applied Mathematics, vol. 46, pp. 133-139, 1967.

[14] G. P. Agrawal, Nonlinear fiber optics, Academic, San Diego, 1989.

[15] M. V. Goldman, "Strong turbulence of plasma waves," Reviews of Modern Physics, vol. 56, no. 4, pp. 709-735, 1984.

[16] M. J. Ablowitz and Z. H. Musslimani, "Integrable Nonlocal Nonlinear Schrödinger Equation," Physical Review Letters, vol. 110, no. 6, 2013.

[17] Y. S. Zhang, D. Qiu, Y. Cheng, and S. J. He, "Rational solution of the nonlocal nonlinear Schrödinger equation and its application in optics," Romanian Journal of Physics, vol. 61, p. 108, 2017.

[18] S.-Y. Lou and H.-Y. Ruan, "Similarity reductions and nonlocal symmetry of the KdV equation," Communications in Theoretical Physics, vol. 25, no. 2, pp. 241-244, 1996.

[19] G. Lévai and M. Znojil, "Systematic search for PT symmetric potentials with real energy spectra," Journal of Physics A: Mathematical and General, vol. 33, no. 40, pp. 7165-7180, 2000.

[20] A. Mostafazadeh, "Pseudo-Hermiticity versus PT symmetry: the necessary condition for the reality of the spectrum of a nonHermitian Hamiltonian," Journal of Mathematical Physics, vol. 43, no. 1, pp. 205-214, 2002.

[21] C. M. Bender, "Making sense of non-Hermitian Hamiltonians," Reports on Progress in Physics, vol. 70, no. 6, pp. 947-1018, 2007.

[22] P. F. Li, D. Mihalache, and L. Li, "Asymmetric solitons in paritytime-symmetric double-hump Scarff-2 potentials," Romanian Journal of Physics, vol. 61, pp. 1028-1039, 2016.

[23] Z. Yan, Z. Wen, and V. V. Konotop, "Solitons in a nonlinear Schrödinger equation with PT-symmetric potentials and inhomogeneous nonlinearity: Stability and excitation of nonlinear modes," Physical Review A, vol. 92, Article ID 023821, 2015. 
[24] M. I. Stockman, "Spaser action, loss compensation, and stability in plasmonic systems with gain," Physical Review Letters, vol. 106, no. 15, Article ID 156802, 2011.

[25] P. Berini and I. de Leon, "Surface plasmon-polariton amplifiers and lasers," Nature Photonics, vol. 6, no. 1, pp. 16-24, 2012.

[26] M. A. Noginov, G. Zhu, A. M. Belgrave et al., "Demonstration of a spaser-based nanolaser," Nature, vol. 460, no. 7259, pp. 11101112, 2009.

[27] Y. D. Chong, L. Ge, and A. D. Stone, "Publisher's Note: ," Physical Review Letters, vol. 108, no. 26, 2012.

[28] Z. Lin, H. Ramezani, T. Eichelkraut, T. Kottos, H. Cao, and D. N. Christodoulides, " Unidirectional Invisibility Induced by ," Physical Review Letters, vol. 106, no. 21, 2011.

[29] V. V. Konotop, J. Yang, and D. A. Zezyulin, "Nonlinear waves in PT-symmetric systems," Reviews of Modern Physics, vol. 88, no. 3, Article ID 035002, 2016.

[30] S. V. Suchkov, A. A. Sukhorukov, J. Huang, S. V. Dmitriev, C. Lee, and Yu. S. Kivshar, "Nonlinear switching and solitons in PT-symmetric photonic systems," Laser \& Photonics Reviews, vol. 10, no. 2, pp. 177-213, 2016.

[31] Y. J. He, X. Zhu, and D. Mihalache, "Dynamics of spatial solitons in parity-time-symmetric optical latties: a selection of recent theoretical results," Romanian Journal of Physics, vol. 61, pp. 595-613, 2016.

[32] V. B. Matveev and M. A. Salle, Darboux Transformations and Solitons, Springer, Berlin, Gemany, 1991.

[33] C. H. Gu, Darboux Transformation in Soliton Theory and its Geometric Applications Shanghai Sci.-Tech, Edu, Publishing House, Shanghai, 2005. 


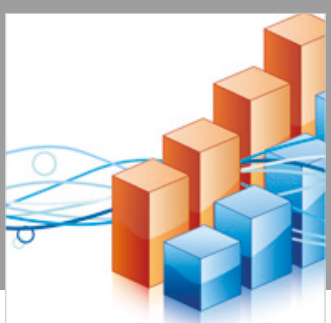

Advances in

Operations Research

\section{-n-m}
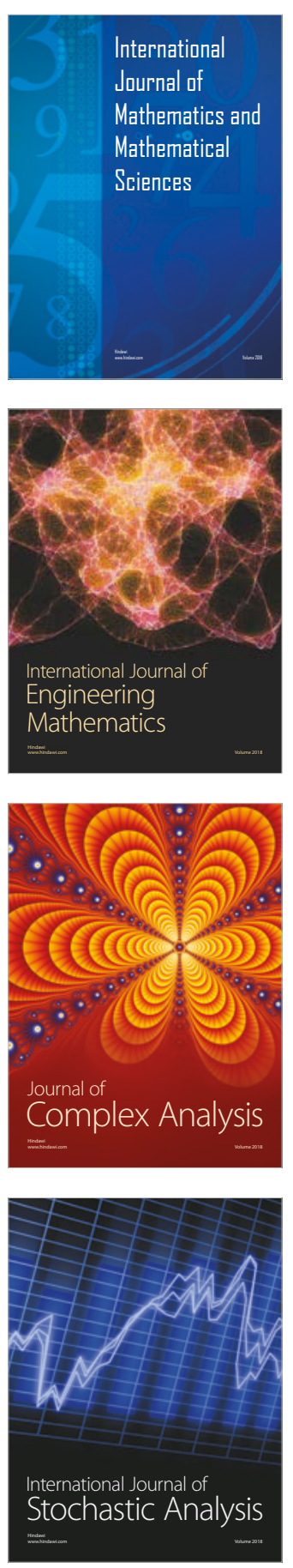
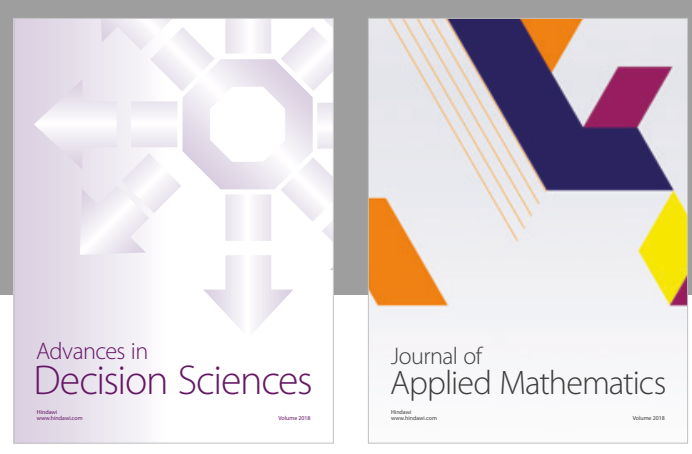

Journal of

Applied Mathematics
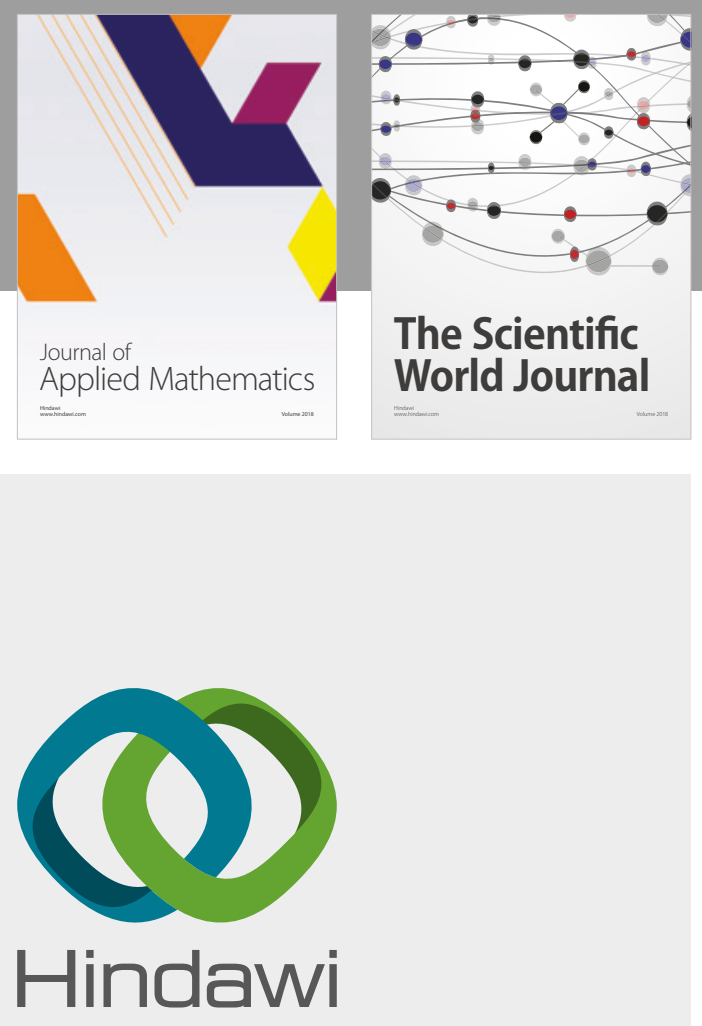

Submit your manuscripts at

www.hindawi.com

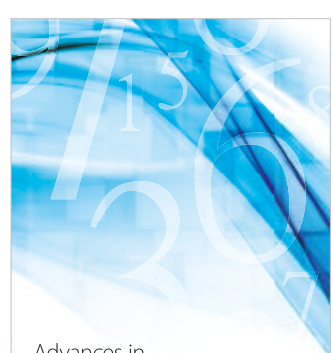

Advances in
Numerical Analysis
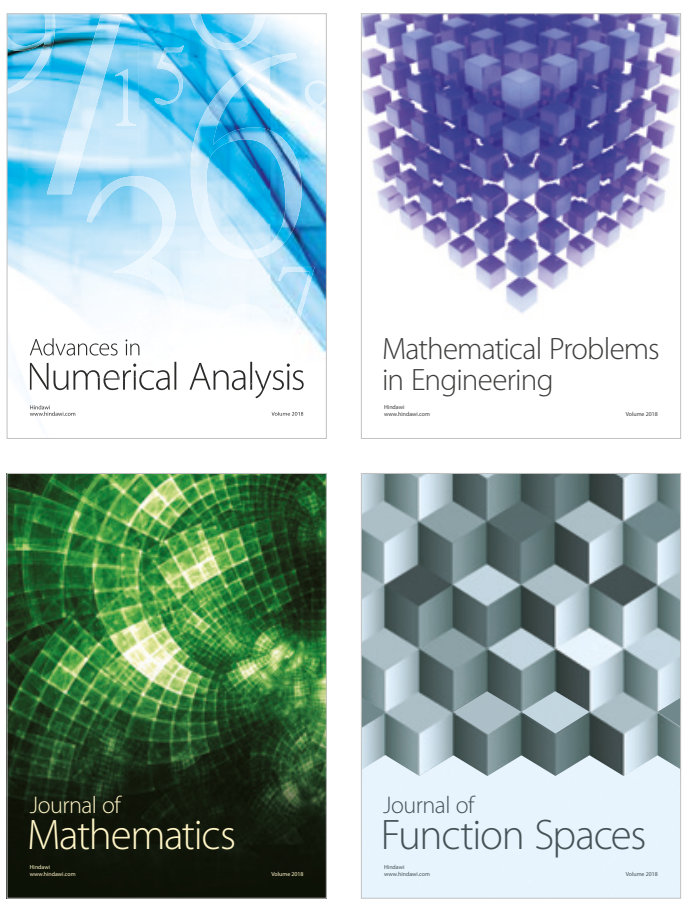

Mathematical Problems in Engineering

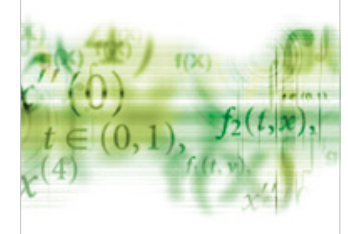

International Journal of

Differential Equations

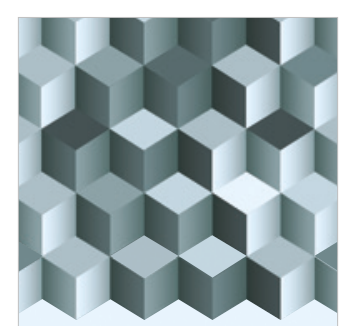

Journal of

Function Spaces

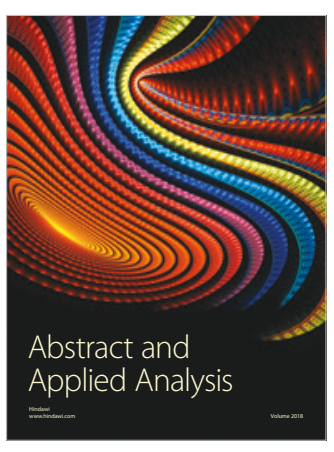

The Scientific

World Journal

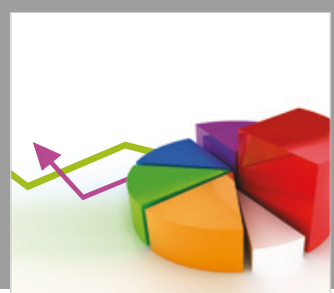

Journal of

Probability and Statistics
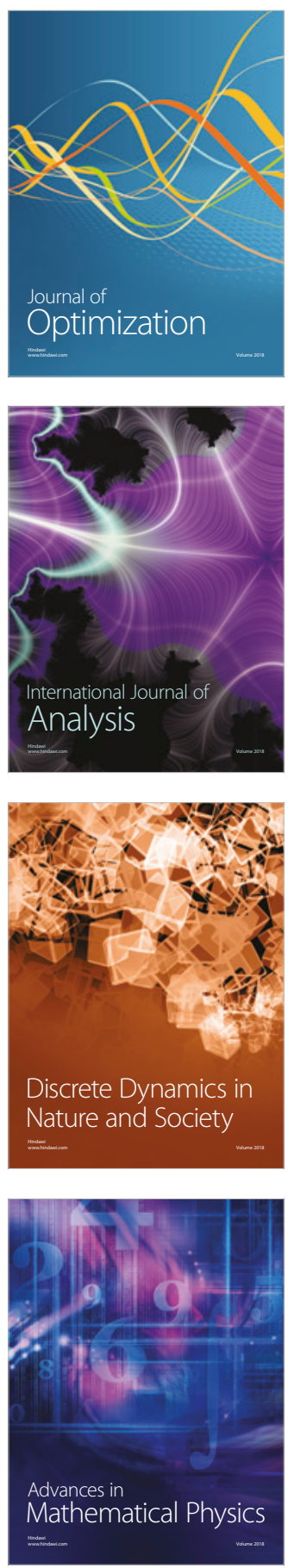